\title{
On the Radio Capacity of TDMA and CDMA for Broadband Wireless Packet Communications
}

\author{
Srikanth V. Krishnamurthy, Member, IEEE, Anthony S. Acampora, Fellow, IEEE, \\ and Michele Zorzi, Senior Member, IEEE
}

\begin{abstract}
Studies of the capacity of cellular systems, stated in terms of the admissible number of remote users, have generally been limited to voice telephony. In this paper, we address the problem of comparing the interference-limited performance of CDMA and TDMA systems in a packet switched environment. The objective is to determine whether the capacity advantages claimed for circuit-switched CDMA still apply in a packet-switched environment, where the natural time diversity of bursty transmission may be a significant factor. Under a set of specific assumptions about the wireless environment (including path loss, shadow fading, multipath delay spread, co-channel interference, power control, coding), we evaluate the number of users which can be admitted to the system while maintaining some desired Qualityof-Service level. Four different classes of users with different characteristics and requirements are considered. The system capacity is found to significantly depend on the QoS objectives, which might be stated in terms of availability of some specified signal to interference level, packet loss rate, or mean tolerable delay. The main finding is that strict requirements imposed on the radio access level tend to favor CDMA, whereas if some form of packet recovery at the higher layers is allowed (implying a relaxed set of requirements on the radio interface), then a somewhat higher capacity may be achieved by TDMA.
\end{abstract}

\section{INTRODUCTION}

Within the field of modern telecommunications, there has recently emerged a strong interest in supporting portable devices capable of wireless communications. Accordingly, Wireless ATM, wherein the concept of bandwidth upon demand is carried over to the wireless world [1]-[2], has enjoyed increasing attention. Seamless extension of ATM from the high bandwidth and low error rate wireline network to the low bandwidth and high error rate wireless network introduces a series of interesting and challenging issues. The harsh fading nature of the wireless channel, and interference due to simultaneous use of the bandwidth by multiple users, cause conditions wherein the intended signal may not be received with satisfactory quality at the base station. While power control may be a partial solution for combating signal loss due to propagation effects, it is also necessary to limit the number of users simultaneously admitted into a system consisting of a cluster of cells in order to keep the interference

S.V. Krishnamurthy is with the Department of Computer Science and Engineering, University of California, Riverside, CA, 92521, e-mail: krish@cs.ucr.edu; A.S. Acampora is with the Department of Electrical and Computer Engineering, University of California, San Diego, La Jolla, CA, 92093, e-mail: acampora@ece.ucsd.edu; M. Zorzi is with the Dipartimento di Ingegneria, Università di Ferrara, Italy, e-mail: zorzi@ing.unife.it. at acceptable levels and to enable hand-off of live connections without unacceptable rates of cell overload or call dropping.

The goal of a call admission control policy is to admit to the system as many users as possible while maintaining the Qualityof-Service (QoS) guarantees of ongoing and incoming calls [3]. The system capacity is then defined as the total number of users that can be admitted to the system. Following a common practice in the literature, we will express this capacity in terms of users per cell, although this number is to be interpreted as an average value since, once a user is admitted to the system, it can roam throughout the service area, and the actual number of users in each cell is a random variable.

Studies of cellular system capacity have been reported in the literature, but most of these have been limited to circuit switched narrow band voice communications [4]-[6]. In [4], the CDMA (Code Division Multiple Access) scheme was compared to static TDMA/FDMA (Time Division Multiple Access/Frequency Division Multiple Access) schemes, and the authors showed that, for telephone traffic, the capability of the CDMA system to tolerate high levels of adjacent cell interference helps in achieving a substantial capacity gain with respect to fixed channel allocation TDMA/FDMA schemes.

This paper complements these studies by considering a wireless network serving constant-bit-rate (CBR) or variable-bit-rate (VBR) users in a packet switched environment. The objective is to estimate and compare the capacity of packet TDMA and packet CDMA approaches. As a first step towards this goal, in this paper we restrict ourselves to a wireless network carrying a single, homogeneous traffic class (i.e., all remotes generate the same type of traffic), and several such single traffic classes are considered. Possible extensions to compute the capacity regions when the network carries multiple traffic classes with different traffic characteristics and QoS requirements can be envisioned but are beyond the scope of this paper.

In an attempt to be thorough, we explicitly account for path loss, shadowing, multipath delay spread and co-channel interference. Further, we investigate the effects of error control coding and power control. We consider convolutional codes of different rates to quantify the effects of error control coding. Two types of power control are considered: (a) coarse power control wherein the power control mechanism compensates only for the pathloss and the shadowing experienced by a user, and (b) fine power control wherein the mechanism is such that it compensates for multipath fading as well. Note that in the latter case the received signal strength due to any remote user's transmission at the base station is constant. Depending on the type of traffic class considered and on the constraints imposed, different 
QoS metrics may be defined. Most of the QoS metrics considered in this work depend on the signal to interference ratio (SIR) at the receiver being greater than a threshold value, fixed a priori. Since closed form expressions for the SIR are difficult to obtain, we use a combination of simulation and analytical models to estimate the statistics of the SIR under various conditions.

In this paper, we are concerned with the radio access performance, i.e., we do not consider higher-layer techniques to recover from transmission errors. In order to draw more definitive conclusions, one must couple such a study with the analysis of protocols at the data-link layer and above. The results we present in this paper provide insight which can lead to formulating meaningful simplified models for the study of these higher-layer issues.

A somewhat general conclusion which can be drawn from our results is that protocols or applications requiring very stringent access performance (e.g., in terms of packet loss rate) would benefit from higher protection as provided by CDMA, whereas protocols and applications which can work in the presence of relatively high packet error rates (e.g., through efficient retransmission error recovery) can accommodate a larger number of users if TDMA mode is chosen. In the environment under consideration, we are able to characterize this trade-off and to identify the CDMA and TDMA cross-over point.

We limit ourselves to the study of the performance of the reverse link (i.e., remote-to-base transmission link). Due to the multiple access nature of the reverse link, one might expect that the forward link performance would be at least as good as that of the reverse link [8]. Ignored throughout are such practical matters as synchronization since we are exclusively interested in comparing the capacities of the two systems as fundamentally limited by propagation effects and multiple access interference.

In Section 2, we present our system model, including propagation effects and allocation strategies used for CDMA and TDMA. Also described in Section 2 are the different classes of traffic considered and the simulation and analytical models. A discussion of the results is presented in Section 3.

\section{System Assumptions}

\section{A Model of the physical layer}

The wireless network is assumed to be divided into regions consisting of contiguous radio cells. Each cell contains a centrally located base-station, and the base stations are deployed in a hexagonal arrangement. For traditional TDMA cellular systems, a seven-cell reuse pattern is often deployed [9]. This results in a 6/7 capacity penalty, which may not be needed in systems where the flexibility of packet switching can be exploited. Therefore, in our work, we assume that the entire bandwidth is reused in every cell for both TDMA and CDMA systems. This may be pessimistic in a TDMA setting, where some interference protection (e.g., via dynamic channel allocation) can help the performance. However, due to the already considerable complexity of the present study, we leave consideration of such schemes for further work.

The propagation model considered throughout accounts for a number of effects. Path loss and shadowing are modeled as the inverse fourth power of the distance, $r^{-4}$, and a log-normal andom variable, $\xi$, respectively, so that the signal power received from a user, averaged over multipath, is proportional to $\beta=r^{-4} \xi$. Based on this quantity, users are assigned to the best base station, i.e., the one with smallest long-term attenuation. This assignment strategy guarantees significantly improved performance with respect to the case of closest base station assignment [10, 11]. The effect of multipath fading [12]-[14] is modeled by means of a complex Gaussian impulse response, $h(t)=h_{i}(t)+j h_{q}(t)$, with exponential multipath intensity profile, i.e., $E\left[h(t) h^{*}(s)\right]=e^{-t / T_{d}}$ if $t=s$ and 0 otherwise. The quantity $T_{d}$ is the delay spread of the channel. As to the second-order statistics of the channel, we consider here for simplicity the block-fading channel model, in which the channel impulse response does not change within a slot, but is independently chosen slot-by-slot. This model also accounts for the effects of user mobility (not explicitly modeled in our MonteCarlo simulations), assumed here sufficient to make successive transmissions experience independent fading conditions. Notice that this assumption of fading independence would also correspond to the use of frequency hopping techniques.

For every packet transmission, we compute, at the receiver, the Signal-to-Interference Ratio (SIR), and assume that the packet is correctly received if and only if the SIR is above a preset threshold, which depends on the details of the physical layer. In particular, the receiver is assumed to consist of a perfectly coherent filter matched to the waveshape produced when a rectangular transmit pulse is "filtered" by the impulse response of the multipath channel ${ }^{1}$. In addition, in the TDMA system, we assume the presence of a zero forcing equalizer which negates the intersymbol interference (ISI) caused by the delay spread of the channel. For both CDMA and TDMA, QPSK modulation is assumed. In order to focus on the interference-limited nature of the systems, thermal noise is neglected throughout.

For a fair comparison, the channel data rate is assumed to be the same for both CDMA and TDMA. In CDMA, the channel data rate is actually the chip rate, with the actual user information rate being $N_{c}$ times slower, where $N_{c}$ is the spreading gain of the CDMA modulation ${ }^{2}$. On the other hand, in TDMA, a user transmits at peak channel rate only during a fraction of the time, resulting again in an actual information rate equal to $1 / N_{c}$ times the peak channel rate. More specifically, we assume a slotted time axis (see Figure 1). Each slot accommodates exactly one TDMA packet. ${ }^{3}$ A frame structure is superimposed, where a frame consists of $N_{c}$ slots. Note that, because of the bandwidth spreading, a CDMA packet will occupy an entire frame (i.e., $N_{c}$ slots).

\section{B Traffic models}

In this paper, we separately and independently consider four traffic classes, representative of a variety of possible services.

Type 1: CBR Traffic. The first class considered is CBR traf-

\footnotetext{
${ }^{1}$ Note that for CDMA this corresponds to an ideal RAKE receiver with an infinite number of fingers.

${ }^{2}$ Note that, strictly speaking, this is true only when no coding is used. If the information sequence is coded before transmission, the effective CDMA spreading gain seen by the encoded stream is reduced according to the code rate, since $N_{c}$ is the ratio between channel rate and user information rate.

${ }^{3}$ In this case as well, if coding is used the slot occupancy of a TDMA packet is increased according to the code rate, so that both information rate and channel symbol rate are kept fixed. Specific explanation is given in Section 2.4.
} 
fic, which may, for example, represent real-time voice or video. For the entire duration of the call, each active CBR user generates packets periodically, at the rate of one packet per frame. Each user is then assigned the same slot in every frame, much like in a circuit-switched system. In this case, interference can be assumed to be persistent (since the same group of users will likely interfere for an extended period of time), and therefore we choose as the QoS metric the probability of outage, i.e., the marginal probability that the SIR is below threshold (in this case, we can assume that the connection will be unacceptably degraded and will therefore be dropped). In the case of speech transmission, packet switching allows discontinuous speech activity to be exploited, through the Speech Activity Detection (SAD) mechanism. In this case, users generate packets only for some fraction of the time (taken to be about $40 \%$ in the numerical results) [4]. In CDMA, this results in a proportionally decreased interference, whereas in TDMA this allows the bandwidth manager to dynamically reallocate slots among active users ${ }^{4}$.

Unlike class 1 , traffic classes 2,3 and 4 consist of bursty packet traffic (VBR). In CDMA, whenever a user sends a packet, the channel is occupied for the entire frame duration, and several overlapping packets may be transmitted in the same frame. In TDMA, a perfect media access protocol is assumed, and the system is able to schedule transmissions so that users in the same cell will transmit in different slots within a frame ${ }^{5}$. Coordination among different cells, though a possibility, is not considered here.

Type 2: Packet Traffic with no retransmissions allowed. Traffic class 2 is a limiting case, with no provision for queueing remote users' packets. Each remote user generates a packet in a frame with probability $q<1$, and that packet is transmitted in the subsequent frame. As before, transmissions for which the SIR is below threshold are unsuccessful, and no retransmission is allowed. Unlike CBR traffic, consecutive randomly generated VBR Class 2 packets encounter different interference power levels since the number of access attempts per slot (TDMA) or frame (CDMA) and the set of interfering users are random. Due to this time diversity feature of packet switching, the fact that the SIR is not adequate during a single packet transmission does not mean that the whole connection will experience poor quality. An appropriate QoS measure for this traffic class is then the probability that the instantaneous packet loss rate exceeds some predetermined value.

Type 3: Packet Traffic with retransmissions allowed and no delay constraints. In traffic class 3, a remote user's queue is assumed to always contain packets awaiting transmission and a user transmits a packet in a frame with probability $q$. If a transmission attempt is unsuccessful, the failed packet is scheduled for retransmission after some random delay. No constraint is imposed on the value of this delay, nor on the number of transmission attempts allowed for a given packet. The remote queue is assumed to be of infinite size, so that, eventually, all packets are successfully delivered if the system is stable. Note

\footnotetext{
${ }^{4}$ We assume here the availability of a perfect bandwidth assignment mechanism, so that the potential of SAD in TDMA can be fully exploited.

${ }^{5}$ Note that this is not possible if the number of active users in a frame exceeds the number of slots. However, in all cases of interest, this event has negligible probability and will be ignored.
}

hat different transmission attempts are subject to different fading/interference effects by virtue of time-diversity. Let the steadystate probability of successful transmission, when, there are (on average) $M$ users per cell, each transmitting with probability $q$, be denoted by $P_{\text {succ }}(M, q)$. The probability that a user successfully transmits in a slot (service rate) is given by $\mu(M, q)=q *$ $P_{\text {succ }}(M, q)$, and, for this case, the maximum value, $\mu_{\max }(M)=$ $\max _{0<q \leq 1}\left(q * P_{\text {succ }}(M, q)\right)$ is the performance metric. The maximum achievable collective throughput per cell (saturation throughput) is then given by

$$
\sigma=\frac{M \mu_{\max }(M)}{N_{c}}
$$

In order to ensure that the system be stable, it is required that the arrival rate to any remote user's queue be less than the achievable service rate, $\mu_{\max }(M)$. Thus, $\sigma$ is the per-cell capacity.

We note here that it is extremely difficult to obtain $P_{\text {succ }}(M, q)$ in a closed form. We use a combination of analysis and simulations to compute the value of $P_{\text {succ }}(M, q)$. For a given value of $\mathrm{M}$ and $\mathrm{q}$, we compute the SIR by analysis (as shown in the following sub-section). We then perform Monte Carlo simulation experiments for an extensive set of channel conditions. In each experiment if a tagged packet under consideration has an associated SIR (as computed by analysis for the particular channel conditions) greater than a pre-requisite threshold, then the packet is deemed successful. By counting the number of successful packet transmissions we can compute the value of $P_{\text {succ }}(M, q)$. In order to compute the saturation throughput, for each value of $\mathrm{M}$, we perform simulations over an exhaustive set of values for $\mathrm{q}$ and estimate the maximum value of $q * P_{\text {succ }}(M, q)$.

Type 4: Packet Traffic with retransmission and delay constraints. Each Type 4 user generates packets according to a Bernoulli arrival process of intensity $q_{i n}$, and stores them in a queue. In any given frame, each remote user with a non-empty queue attempts to transmit a packet with probability $q$. The steady state probability of a packet being successfully transmitted is $P_{s u c c}(M, q)$. If the transmission attempt fails, the packet is returned to the queue for retransmission. The performance metric defined for traffic class 4 is the mean delay experienced by a packet, which includes the queueing delay as well as delay due to failed transmission attempts. Note that due to the time diversity feature of packet switching (which randomizes the interference in successive transmission attempts) and under the assumption of fine power control (which basically removes the propagation effects seen by any single user), successive transmission attempts can be approximated as independent and identically distributed with success probability $P_{\text {succ }}(M, q)$. Accordingly, each remote user's queue behaves as an $\mathrm{M} / \mathrm{M} / 1$ queue. Let $P_{0}$ be the probability that a given remote user's queue is empty. If there are $M$ users in the network, each would transmit with an effective rate of $q^{\prime}=q\left(1-P_{0}\right)$ packets per frame. In order for an $M / M / 1$ queue to be stable, the average interarrival time must be greater than the average service time. Consequently it is necessary that $q_{i n}$ (input arrival rate as defined earlier) be less than $q P_{s u c c}\left(M, q^{\prime}\right)$. The mean delay experienced by a packet when $M$ users are present in the system 
is then given by [15]

$$
\bar{D}(M)=\frac{1}{q P_{\text {succ }}\left(M, q^{\prime}\right)-q_{i n}} .
$$

In order to minimize the mean delay, for a given input rate of $q_{i n}$, the average service rate $q P_{\text {succ }}\left(M, q^{\prime}\right)$ must be maximized with respect to $q$, taking into account that $q^{\prime}=q\left(1-P_{0}\right)$ and that the probability that a given remote user's queue is empty is given by

$$
P_{0}=1-\frac{q_{i n}}{q P_{s u c c}\left(M, q^{\prime}\right)}
$$

\section{SIR analysis}

Let there be $J+1$ remote users, uniformly distributed in the cluster of cells forming the region of interest. Let us focus on the transmission of a given user (say the 0-th user) in the center cell, so that edge effects are avoided. Let $r_{k}$ be the distance between the $k$-th transmitter in the cluster and the considered base station. Furthermore, let $\xi_{k}$ and $h_{k}(t)$ be the shadowing attenuation and the multipath impulse response affecting the user's signal, respectively. Let $p(t)$ be a rectangular pulse of unit amplitude and duration $T_{c}$, where $T_{c}$ is the channel symbol (chip) duration.

In Appendix A we show that for CDMA with neither coding nor power control, the SIR with which a packet is received is given by:

$$
S I R_{C D M A}=\frac{r_{0}^{-4} \xi_{0} N_{c}^{2} F^{2}(0)}{I_{C D M A}} .
$$

In the above equation $I_{C D M A}$ is the interference experienced by the packet due to $J$ simultaneously transmitting interferers and a self noise component (due to frequency selective fading) and is given by:

$$
\begin{gathered}
I_{C D M A}=\left\{4 r_{0}^{-4} \xi_{0} \sum_{i=1}^{N_{c}-1}\left(N_{c}-i\right) F^{2}(i)\right. \\
\left.+\sum_{k=1}^{J} r_{k}^{-4} \xi_{k} \sum_{i=-\left(N_{c}-1\right)}^{N_{c}-1}\left(N_{c}-|i|\right) G_{k}^{2}(i)\right\}
\end{gathered}
$$

where $F(j)=h_{0}(t) * p(t) * h_{0}(t) * p\left(t-j T_{c}\right)$ and $G_{k}(j)=$ $h_{0}(t) * p(t) * h_{k}(t) * p\left(t-j T_{c}\right)$.

In Appendix B, we show that under the same conditions, the SIR for TDMA is

$$
S I R_{T D M A}=\frac{r_{0}^{-4} \xi_{0} F^{2}(0)}{I_{T D M A}} .
$$

As before, $I_{T D M A}$ represents the interference experienced by the packet in the TDMA system and is given by:

$$
\begin{array}{r}
I_{T D M A}=\sum_{m=1}^{J} r_{m}^{-4} \xi_{m} \sum_{k=-K}^{K} C_{k} \sum_{l=-K}^{K} C_{l}\{ \\
\left.\sum_{i=-K}^{K} G_{m}(k+i) G_{m}(l+i)\right\},
\end{array}
$$

where $C_{k}, k=-K, \ldots, K$, represent the tap weights of the zero forcing equalizer.

Equations (4) and (6) provide the conditional SIR, given the number of interferers, $J$, and the propagation conditions of all users. These analytical expressions can be used in a Monte Carlo simulation for the evaluation of the desired performance metrics.

Coding may be used in both the CDMA and TDMA systems. In a CDMA system, use of a convolutional code of rate $k / n$ would reduce the spreading gain by a factor of $n / k$, so that both the information rate and the channel chip rate are unchanged. In a TDMA system, coding would result in a packet occupying $n / k$ slots instead of just one. Coding allows achievement of a given bit error rate objective with lower SIR threshold. This may be represented as a coding gain, which is the difference in SIR needed by coded and uncoded systems to obtain the same BER performance.

In all our computations, we consider the SIR of a code symbol (as opposed to the SIR of an information bit) for making a decision on the success/failure of a packet. An information bit consists of $n / k$ code symbols when a code of rate $k / n$ is used. At the output of the correlator, the signal powers of the $n / k$ code symbols in the information bit add up coherently, while the powers interfering with those code symbols add up incoherently. This yields an additional coherence gain of $10 \log _{10}(n / k)$ $\mathrm{dB}$. Since convolutional error control codes provide better immunity to independent errors than to correlated errors, the coded performance is expected to improve when interleaving is used [8], whereby bits are scrambled among packets prior to transmission, so that the correlation between errors is reduced. The price to be paid in this case for the improved coding performance is a delay increase.

Power control schemes are used to compensate for time variation in signal strength arising from various propagation phenomena. Open loop or coarse power control compensates only for the path loss and shadowing experienced by a remote's signal. Thus, in the absence of multipath effects, the signals received from all simultaneously transmitting users within a cell will be of the same power (perfect power control will be assumed throughout). If fine or closed loop power control is implemented, a remote will adjust its transmit power such that, irrespective of its position and the associated shadow and multipath fading, the power of the intended signal component at the base-station is a constant ${ }^{6}$. The power control considered in this paper is strength-based, i.e., it achieves constant received power. Another form of power control, whose objective is to equalize the SIR at the receiver, may be more favorable to TDMA, but may also suffer from stability problems, and will not be considered here.

\section{RESUlts AND Discussion}

In this section, we present some results which are representative of the performance of the CDMA and TDMA systems. We do not attempt an exhaustive performance evaluation of the two schemes, but rather focus on a comparison of their radio access capacity. Our results, generated according to the analyt-

\footnotetext{
${ }^{6}$ It should be noted that this does not completely eliminate multipath effects i.e., time dispersion will still be present.
} 
ical/simulation technique described in the previous section, are presented in terms of QoS metric (which is different for different traffic classes) vs. the average number of admitted users per cell. As a general conclusion, we observed that the capacity assessment of TDMA and CDMA significantly depends on the QoS metrics chosen and on the QoS requirements. Typically, CDMA better satisfies strict QoS constraints (i.e., small packet error rates), due to its inherent ability to tolerate some degree of interference while still providing good quality. On the other hand, TDMA is more sensitive to moderate or high levels of interference, and therefore in this context it offers a higher capacity when less stringent requirements are placed on the physical/access layer. It should be noted that this does not mean that TDMA cannot be used for high-quality communications, since time diversity coupled with the short durtion of the TDMA packets allows for the recovery at higher layers (e.g., data-link) of those packets which are lost on the radio interface. The study of these higher-layer protocols is, however, beyond the scope of the present paper, but our results may be applicable to such a future study.

In the simulations, we assume that satisfactory signal quality can be achieved with a SIR of $8 \mathrm{~dB}$ (computed after the frontend processing, including despreading, decoding and equalization as applicable). The standard deviation of the lognormal shadowing is assumed to be $8 \mathrm{~dB}$. To fully capture interference effects, the geographical region under consideration is a cluster of 61 cells. The use of error-control codes is assumed to yield asymptotic coding gains. In particular, for convolutional codes with soft decision decoding, the coding gains assumed are $7 \mathrm{~dB}$ for a rate $1 / 2$ code, $7.3 \mathrm{~dB}$ for a rate $1 / 3$ code, and $7.4 \mathrm{~dB}$ for a rate $1 / 4$ code, respectively [16]. If hard decision decoding is used, the coding gain is about $3 \mathrm{~dB}$ smaller. The constraint length for each of these codes is 7 . We also considered a rate $2 / 3$ code with a constraint length of 4 for equivalent decoder complexity. For this code, the asymptotic coding gain is 5.2 $\mathrm{dB}$ with soft decision decoding. Finally, before we discuss our results we list again, some of the primary assumptions that we make $^{7}$.

- We assume a frequency reuse factor of one for both CDMA and TDMA systems.

- We assume two types of power control. Coarse power control compensates for pathloss and shadowing. Fine power control compensates for multipath fading as well.

- We assume that a zero forcing equalizer is used with the TDMA system and that this equalizer completely eliminates time dispersion for the desired signal.

- In TDMA, a perfect medium access control protocol is present. This protocol allows transmissions to be scheduled such that users within a cell transmit in different timeslots within a frame.

- We ignore synchronization issues

We note that some of these assumptions affect the numerical results presented, and such effects may be different for TDMA and CDMA. On the one hand, note that some assumptions favor CDMA (e.g., perfect synchronization) while others favor TDMA (e.g., perfect MAC). On the other hand, the main find-

\footnotetext{
${ }^{7}$ These assumptions were described earlier in Section 2.
}

ings of this study, e.g., the fact that the relative performance of CDMA and TDMA depend on the QoS specifications and on the type of traffic, is still qualitatively true. Therefore, although a more precise assessment of this comparison should take into account more details, what is presented here is a valuable first step towards a fuller understanding of the issues involved.

\section{A Results for CBR traffic}

We present first some results for the Type 1 traffic, i.e., CBR traffic. Figure 2 illustrates the effects of power control on both TDMA and CDMA, for $N_{c}=50$ and $T_{d}=2 T_{c}$ (unless otherwise noted, these parameters will be used for all results in the following). It can be clearly seen that, as expected, CDMA benefits from the use of fine power control, compared to the case of coarse power control (performance with no form of power control is not considered for CDMA). On the other hand, TDMA shows a very robust behavior with respect to the type of power control used, with essentially the same performance in both cases, which in turn is significantly better than no power control at all. This can be explained by the fact that TDMA packet successes mostly occur when there is almost no interference, in which case the performance depends more on proper equalization than on the intended signal power. We also studied the effect of changing the value of $N_{c}$ (not shown in the Figure): as expected, by doubling $N_{c}$, one can approximately admit twice as many users, so that results for higher values of $N_{c}$ can be similarly extrapolated $^{8}$. Note, however, that increasing $N_{c}$ requires that either the information rate be decreased or the bandwidth be increased. Also, consistent with [4, 7], the results shown in Figure 3 confirm that the use of SAD produces approximately a capacity 2.5 times larger (for a speech activity factor of $40 \%$ ).

Figure 4 illustrates the effects of using convolutional codes of different rates in a CDMA system. No SAD is considered here, but a good approximation for the case with SAD can be derived by appropriately scaling the results shown. It is seen that choosing a code rate smaller than $1 / 2$ does not result in any further significant improvement, due to similar values of the coding gain ${ }^{9}$. In the rest of the paper, a rate $1 / 2$ code will therefore be used.

In Figure 5 (also with no SAD), we present a direct comparison between TDMA and CDMA for the CBR traffic class for some specific conditions. The curves can be seen to cross at some critical value of the outage probability (e.g., about 0.02 when a rate $1 / 2$ code is used). CDMA allows more users to be admitted than TDMA when the constraint on the outage probability is smaller than this critical value, whereas if outage probabilities larger than the critical value are permitted, TDMA provides higher capacity. The CDMA system can easily enjoy the benefits of SAD, so that the capacity results as given in Figure 5 can be multiplied by a factor of 2.5 . For an outage probability of less than 0.01 , results indicate that about $13 \simeq N_{c} / 4$ users may be admitted to the CDMA system. The performance benefit provided by SAD and cell sectorization cannot be as easily achieved in circuit-mode TDMA. However, if one is willing to accept the additional complexity of dynamic slot reassignment,

\footnotetext{
${ }^{8}$ Note that this may not be true for smaller values of $N_{c}$, where the behavior is not linear

${ }^{9}$ Note that, as the code rate is decreased, the coherence gain due to the fact that coded symbols combine coherently while noise combines incoherently is counterbalanced by the corresponding loss in processing gain seen by the coded sequence.
} 
the same capacity gains can be achieved for both TDMA and CDMA.

The fact that the curves for CDMA and TDMA cross at some point is an important result, and being able to quantitatively identify that critical point and to study its sensitivity to the various environmental factors may be key for efficient design of the access scheme. For example, we found that the crossing point moves to the right (i.e., the regime over which CDMA provides higher capacity increases) if the frequency selectivity of the channel increases. In fact, the performance of the CDMA scheme improves with an increase in delay spread, since this translates into greater received signal power because there are then more resolvable paths (recall that we assume here a perfectly matched filter at the receiver, which can resolve all paths). On the other hand, the performance of TDMA is relatively insensitive to the value of $T_{d}$, since the effect of the delay spread in this case (i.e., the ISI) is removed by the equalizer ${ }^{10}$.

\section{B Results for VBR traffic}

In this subsection, we present results for the three VBR classes as described in Section 2.

The QoS measure considered for Traffic Class 2 (VBR traffic with no queueing permitted at the remote) is the probability that the packet loss rate exceeds some maximum tolerable value. The packet loss rate suffered by any user is dependent on the interference experienced by that user. The interference in turn depends on the propagation conditions of all simultaneously transmitting users. In Figure 6, we have considered two values for the maximum tolerable packet loss rate, i.e., $1 \%$ and $10 \%$. We remark once again that the packet loss considered here is observed at the radio access level and, while certainly affecting the performance of protocols and applications at higher layers, it is not representative of the quality perceived by the user. In other words, this study is mostly concerned with lower-layer performance, whereas a higher-layer study based on the results here presented still needs to be addressed in order to fully characterize the QoS enjoyed by the applications.

The sensitivity of the CDMA system to the value of the tolerated packet loss rate is relatively small due to the fact that spread spectrum provides good protection against moderate interference. For typical values of SIR, stricter requirements of no more than $1 \%$ packet loss are usually satisfied, whereas under heavy interference conditions, CDMA breaks down and cannot meet even looser loss requirements of $10 \%$. On the other hand, the outage probability for TDMA is very dependent on the threshold packet loss objective; for our range of parameters, a $10 \%$ objective can be met whereas a $1 \%$ objective cannot.

Figure 6 shows the probability of outage, i.e., the probability that the instantaneous packet loss probability exceeds a specified value, denoted by $x$ in the graph labels ${ }^{11}$. Consistent with the above discussion, Figure 6 shows that, if the packet loss rate must be $1 \%$ or lower, CDMA in general provides higher capacity (if we want a packet loss rate of no more than $1 \%$ at least $90 \%$ of the time, when a rate $1 / 2$ code is used, CDMA provides

\footnotetext{
${ }^{10}$ For very large values of the delay spread, the performance of TDMA will be affected by interference enhancement in the equalizer, leading to degraded SIR.

${ }^{11}$ The value of the outage probability may be interpreted as the long-term average of the fraction of time a user experiences a packet loss rate in excess of $x$, or equivalently the fraction of users experiencing the same condition throughout the system.
}

more than twice the capacity of TDMA), whereas the opposite might be true if the QoS requirement is relaxed to allow packet loss rates of up to $10 \%$. A possible way to improve matters for TDMA might be to reintroduce some degree of frequency isolation, e.g., not reusing the same spectrum in all cells. We believe that a more efficient system strategy would be to accept relatively high packet loss rates at the access layer, coupled with some smart scheduling strategies for efficient retransmission, as was proposed in [7]. Another alternative might be to dynamically assign channels among different cells, which involves considerably more complexity but can potentially prevent worstcase occurrences, thereby greatly improving the overall performance. Finally, more advanced signal processing techniques (e.g., smart antenna arrays) can also be used to reduce the effect of interference. Due to the already considerable complexity of our physical-layer study, we have not addressed these aspects, which are left for future efforts. We make a note here that although the chosen value of the spreading gain $N_{c}$ is 50 , the number of admitted users could be much higher since the user activity $q$ is small (equal to $10 \%$ in this case).

Figures 7 illustrates the performance of the TDMA and CDMA systems with Type 3 traffic, i.e., packet traffic users in heavy load. Plotted as a function of the expected number of users per cell is the saturation throughput, which is the amount of traffic that the network can carry with no delay constraints. For a given number of admitted users, $M$, and a given transmit probability $q$, the probability of success $P_{\text {succ }}(M, q)$ is found by simulation. Note that, in computing this probability of success, all possible channel conditions are considered. The service rate $q P_{\text {succ }}(M, q)$ is then maximized with respect to the transmit probability $q$. The value of $q$ which achieves this maximum also achieves the saturation throughput as explained in Section 2. It is to be noted that, since the metrics thus computed are averages (the averaging being done over all possible channel conditions), there are no QoS objectives defined for this traffic class. The effects of various parameters on the CDMA system are shown in Figure 7. From Figure 7, it is seen that the TDMA system achieves a higher saturation throughput and a better maximum achievable service rate than does the CDMA system. This is not surprising since, without any delay constraints, retransmission with time diversity is expected to be more bandwidth efficient than the a priori protection provided by bandwidth spreading [7] (note, in fact, that in CDMA, bandwidth is spread even when it is not needed, i.e., when there is little interference).

In Figure 7, it is seen that the TDMA throughput first increases with the number of users per cell, $M$, but then asymptotically decreases as $M$ becomes large. This is due to the fact that there is no intra-cell contention among the TDMA users for low values of $M$. However, as $M$ increases, a user's signal would be subject to intra-cell interference in addition to inter-cell interference, causing an overall throughput reduction. Thus, when the per-user transmission probability is chosen to maximize the saturation throughput, adding users eventually causes an asymptotic reduction in throughput.

Finally, Figure 8 shows results of mean delay for VBR Type 4 traffic. When analyzing the performance of this traffic class, we assumed that the co-channel interference and multipath fading experienced by a packet vary independently from one transmis- 
sion attempt to another. As discussed in Section 2, this may be a reasonable assumption if fine power control is used and in the presence of a sufficient degree of randomization of the transmission attempts. The mean delay is minimized with respect to the transmit probability $q$. From queueing theory, the minimum mean delay is achieved when $q$ is chosen so as to maximize the throughput. Thus, as in the case of Type 3 traffic, one might expect the TDMA mean delay performance to be better than that of CDMA. Figure 8 compares the mean delay performance of CDMA and TDMA. It is seen that a TDMA scheme using a rate $1 / 2$ code can admit approximately twice as many remote-users as may be admitted in a CDMA scheme using a rate $1 / 2$ code before the system becomes unstable. Other results, not shown here, indicate that reducing the arrival rate to a remote user's queue, $q_{i n}$, by half approximately doubles the number of admissible users, as expected.

\section{CONCLUSIONS AND FUtURE WORK}

In this work we have compared the performances of CDMA and TDMA systems in a packet switched wireless network. Different traffic classes, requiring different QoS metrics, have been independently considered. By means of analysis and simulations, the capacity of the network, stated in terms of the maximum number of users admissible to a geographical region such that some QoS objective can be guaranteed, was found. Results obtained under a specific set of assumptions show that the relative performance of CDMA and TDMA, expressed in terms of admissible number of users, largely depends on the QoS objectives set at the radio access level. As a general result, TDMA may be used in conjunction with protocol stacks which are able to recover from relatively high packet loss rates on the air interface, whereas CDMA should be used when small packet error rates at the radio access level are necessary.

While the results obtained in this work provide some valuable insight about the performance of the TDMA and CDMA schemes in a wireless packet environment, further study is required in order to draw more firm conclusions while also relaxing some of the simplifying assumptions made here. In particular, extending the effect of this study to higher layers in the protocol stack (e.g., data-link error control) may enable a clearer assessment of the performance of the whole system. Also, this work is limited to the independent study of different traffic classes. Study of co-existing multiple traffic classes with differing QoS requirements needs further attention. Also, use of sectorized or array antennas, which may yield significant performance improvements in both TDMA and CDMA, also merits future investigation.

Finally, while the objective of the paper was to compare CDMA with TDMA with a frequency re-use factor of 1 , we recognize that a comparison with multiple frequency TDMA may be appropriate. However, it is beyond the scope of this preliminary work. We point out that since we have compared the schemes with a varying number of users, using multiple frequencies (or using frequency hopping) will cause the interference that is experienced by a transmitting user due to simultaneously transmitting interferers to be reduced. This may be represented as a reduction in the number of interfering users or, equivalently, as a capacity increase. However, we note that this capacity increase (in terms of number of users per cell) is actually achieved at the expense of a capacity loss due to the use of a frequency reuse plan. In a packet-switched situation it is likely that not using full frequency reuse will lead to worse results.

\section{A Estimation OF SIR IN THE CDMA SYSTEM}

\section{A Signal Component and Self Interference}

Let a single user, transmit a signal given by:

$$
s_{0}(t)=\sum_{k=0}^{N_{c}-1} a_{k} p\left(t-k T_{c}\right)
$$

which passes through a channel with a complex impulse response $h_{0}(t)=h_{0 i}(t)+j h_{0 q}(t)$. Let $\rho_{0}(t)$ denote the input to the receiver (i.e., $\rho_{0}(t)=s_{0}(t) * h_{0}(t)$ ), which consists of a matched filter with impulse response $\rho_{0}^{*}(-t)$, and let $y_{0}(t)$ be the output of the matched filter, given by $y_{0}(t)=$ $\rho_{0}(t) * \rho_{0}^{*}(-t) .=y_{\text {inphase }}(t)+j y_{\text {quadrature }}(t)$.

The output of the matched filter is sampled at time $t=0$ :

$$
\begin{aligned}
& y_{0}(0)=y_{I}(0)+y_{Q}(0) \\
& =\int_{-\infty}^{\infty}\left\{\sum_{k=0}^{N_{c}-1} a_{k} p\left(t-k T_{c}\right) * h_{0 i}(t)\right\}^{2} \\
& +\left\{\sum_{k=0}^{N_{c}-1} a_{k} p\left(t-k T_{c}\right) * h_{0 q}(t)\right\}^{2} d t .
\end{aligned}
$$

Also,

$$
\begin{array}{r}
y_{I}(0)=\int_{-\infty}^{\infty} \sum_{k=0}^{N_{c}-1} a_{k}^{2}\left[f_{i}\left(t-k T_{c}\right)\right]^{2} d t+ \\
\sum_{k=0}^{N_{c}-1} \sum_{\substack{j=0 \\
j \neq k}}^{N_{c}-1} a_{k} a_{j} \int_{-\infty}^{\infty} f_{i}\left(t-j T_{c}\right) f_{i}\left(t-k T_{c}\right) d t
\end{array}
$$

where, $f_{i}\left(t-k T_{c}\right)=\int_{-\infty}^{\infty} p\left(\alpha-k T_{c}\right) h_{0 i}(t-\alpha) d \alpha$. Denoting $\int_{-\infty}^{\infty}\left[f_{i}\left(t-j T_{c}\right) f_{i}\left(t-k T_{c}\right)\right] d t$ by $F_{i}(j-k)$, we get:

$$
y_{I}(0)=N_{c} F_{i}(0)+2 \sum_{k=0}^{N_{c}-2} \sum_{j=k+1}^{N_{c}-1} a_{j} a_{k} F_{i}(j-k) .
$$

Using a subscript $q$ to represent equivalent terms for the quadrature channel, Equation (11) can be written as:

$$
\begin{gathered}
y_{l}(0)=N_{c}\left(F_{i}(0)+F_{q}(0)\right) \\
+2 \sum_{j=1}^{\left(N_{c}-1\right)} \sum_{j=k+1}^{N_{c}-1} a_{j} a_{k}\left(F_{i}(j-k)+F_{q}(j-k)\right)
\end{gathered}
$$

Notice that all the terms in the above expression are real and hence, $y_{0}(0)$ is a real quantity. The notation $F(j)=F_{i}(j)+$ $F_{q}(j)$ is used hence forth. The first term of Equation (12) represents the signal component and the second term represents self noise due to multipath propagation. Since $a_{j}$ and $a_{k}$ take the values +1 and -1 with equal probability and are assumed to be statistically independent of each other (ideal spread spectrum 
modulation), the expected value of the self noise is zero, and its variance is given by:

$$
\overline{n^{2}}=4 \sum_{j=1}^{N_{c}-1}\left(N_{c}-j\right) F^{2}(j) .
$$

Note that, $F(j)$ is a deterministic quantity, since, in this analysis, $h_{0}(t)$ is a known, single sample from the random process $\left\{h_{0}(t),-\infty<t<\infty\right\}$.

\section{B Multiple Access Interference}

In this section, we compute the multiple access interference due to simultaneously transmitting users. For simplicity, we omit subscripts and complex notations. Let us consider an interfering user, say user $u$, whose signal may be represented as:

$$
z(t)=\sum_{k=0}^{N_{c}-1} b_{k} p\left(t-k T_{c}\right)
$$

Let the impulse response of the channel through which this signal passes be $h_{z}(t)$. Then, arriving at the input to the receiver is:

$$
w(t)=z(t) * h_{z}(t) .
$$

Let $\eta(t)$ be the interference caused by user $u$ at the output of the receiver filter, which is matched to the signal from user 0 . Then, at the sample time,

$$
\begin{aligned}
\eta(0)= & \int_{-\infty}^{\infty} \int_{-\infty}^{\infty} \int_{-\infty}^{\infty} \sum_{j=0}^{N_{c}-1} \sum_{k=0}^{N_{c}-1}\left\{b_{j} a_{k} p\left(\beta-j T_{c}\right)\right. \\
& \left.p\left(\gamma-k T_{c}\right) h_{z}(\alpha-\beta) h_{0}(\alpha-\gamma)\right\} d \beta d \gamma d \alpha
\end{aligned}
$$

Since $E\left(a_{k}\right)=E\left(b_{j}\right)=0$ and $a_{k}$ and $b_{j}$ are statistically independent, $\bar{\eta}=0$.

In a manner similar to the definition of $f(t)$ let us define:

$$
g_{u}\left(t-k T_{c}\right)=\int_{-\infty}^{\infty} p\left(\beta-k T_{c}\right) h_{z}(t-\beta) d \beta
$$

Further, let

$$
G_{u}(k-j)=\int_{-\infty}^{\infty} f\left(\alpha-k T_{c}\right) g_{u}\left(\alpha-j T_{c}\right) d \alpha .
$$

Then, we write Equation (16) as:

$$
\eta(0)=\sum_{j=0}^{N_{c}-1} \sum_{k=0}^{N_{c}-1} b_{j} a_{k} G_{u}(k-j)
$$

and it can be shown (with some algebraic simplifications) that

$$
\overline{\eta^{2}(0)}=\sum_{j=-\left(N_{c}-1\right)}^{N_{c}-1}\left(N_{c}-|j|\right) G_{u}^{2}(j)
$$

Let there be $J$ multiple access interferers in the region of interest. Let $r_{u}$ be the distance of the $u$ th interferer from the base-station of the intended user, and $\xi_{u}$ is the lognormal shadowing of the $u$ th interferer with respect to the base-station of the intended user; $r_{0}$ and $\xi_{0}$ are the corresponding parameters of the intended user. Then, the interference experienced by the intended user both due to self noise and due to the $J$ interferers is given by:

$$
\begin{array}{r}
I_{C D M A}=4 r_{0}^{-4} \xi_{0} \sum_{i=1}^{N_{c}-1}\left(N_{c}-i\right) F^{2}(i) \\
+\sum_{u=1}^{J} r_{u}^{-4} \xi_{u} \sum_{i=-\left(N_{c}-1\right)}^{N_{c}-1}\left(N_{c}-|i|\right) G_{u}^{2}(i)
\end{array}
$$

Then, the SIR seen by the intended user, in the presence of $J$ multiple access interferers, can be expressed as:

$$
S I R=\frac{r_{0}^{-4} \xi_{0} N_{c}^{2} F^{2}(0)}{I_{C D M A}} .
$$

\section{B Estimation Of SIR IN THE TDMA SYSTEM}

Let a given user, transmit a signal such that a single bit from this user is given by:

$$
s_{0}(t)=a_{0} p(t)
$$

iLet $\rho_{0}(t)$ denote the input to the receiver. Then,

$$
\rho_{0}(t)=s_{0}(t) * h_{0}(t),
$$

where $h_{0}(t)=h_{0 i}(t)+j h_{0 q}(t)$ is the impulse response of the complex Gaussian channel through which the signal passes.

The receiver is assumed to consist of a matched filter whose impulse response is given by $\rho_{0}^{*}(-t)$. The output of the matched filter is sampled at time $t=0$. The output consists of the signal $s_{0}(t)$, the multiple access interference, and an intersymbol interference (ISI) contribution from bits that precede and follow $s_{0}(t)$. At the output of the matched filter, let the response of our given user at the output of the matched filter, (consisting of the intended signal and the ISI) be denoted by $y_{0}(t)$. Then, at the sample time,

$$
\begin{gathered}
y_{0}(0)= \\
\int_{-\infty}^{\infty}\left[\sum_{i=-K}^{K} a_{i} p\left(t-i T_{b}\right) * h_{0}(t)\right] \cdot\left[p(t) * h_{0}^{*}(t)\right] d t
\end{gathered}
$$

Expanding Equation (24), we get:

$$
y_{0}(0)=a_{0} \int_{-\infty}^{\infty}\left[f_{i}^{2}(t)+f_{q}^{2}(t)\right]+
$$

$$
\begin{aligned}
& \sum_{\substack{i=-K \\
i \neq 0}}^{K} a_{i}\left\{\int_{-\infty}^{\infty} f_{i}\left(t-i T_{b}\right) f_{i}(t)+f_{q}\left(t-i T_{b}\right) f_{q}(t) d t\right. \\
& \left.\quad+j \int_{-\infty}^{\infty} f_{i}\left(t-i T_{b}\right)+f_{q}(t)-f_{q}\left(t-i T_{b}\right) f_{i}(t) d t\right\}
\end{aligned}
$$

where

- $T_{b}$ is the bit time. 
- $f_{i}\left(t-i T_{b}\right)=\int_{-\infty}^{\infty} p\left(\psi-i T_{b}\right) h_{0 i}(t-\psi) d \psi$

- $f_{q}\left(t-i T_{b}\right)=\int_{-\infty}^{\infty} p\left(\psi-i T_{b}\right) h_{0 q}(t-\psi) d \psi$

- $K$ is the maximum number of bits which contribute to the intersymbol interference. This number $K$ is a function of the delay spread of the channel.

We are interested in only real part of the signal appearing at the output of the receiver. It can then be shown that the signal power is given by

$$
\left(F_{i}(0)+F_{q}(0)\right)^{2}=F^{2}(0)
$$

where,

$$
\begin{aligned}
& F_{i}(k)=\int_{-\infty}^{\infty} f_{i}(t) f_{i}\left(t-k T_{b}\right) d t \text { and }, \\
& F_{q}(k)=\int_{-\infty}^{\infty} f_{q}(t) f_{q}\left(t-k T_{b}\right) d t .
\end{aligned}
$$

The ISI noise component is given by

$$
n=\sum_{\substack{i=-K \\ i \neq 0}}^{K} a_{i} F(i)
$$

Note that the mean value of the $I S I$ is zero. It can be shown that the variance of the ISI is given by:

$$
E\left(n^{2}\right)=2 \sum_{i=1}^{K} F^{2}(i)
$$

Since, for the signal, we are considering a known channel impulse response (as in the CDMA case), $F(i)$ is deterministic.

To calculate the multiple access interference, we denote user $u$ 's signal by

$$
s_{u}(t)=\sum_{i=-K}^{K} b_{i} p\left(t-i T_{d}\right)
$$

and the impulse response of the channel through which this signal passes by $h_{u}(t)$. It can then be shown that total the multiple access interference due to the presence of $J$ interfering users is given by ${ }^{12}$

$$
E\left(\eta^{2}\right)=\sum_{u=1}^{J} \sum_{i=-K}^{K} G_{u}^{2}(i)
$$

where $\eta$ is the random variable representing the multiple access interference.

Using Equations 25, 26 and 27, and by considering the effects of pathloss and shadowing, the SIR can be expressed as:

$$
\begin{gathered}
S I R= \\
\frac{r_{0}^{-4} \xi_{0} F^{2}(0)}{2 r_{0}^{-4} \xi_{0} \sum_{i=1}^{K} F^{2}(i)+\sum_{u=1}^{J} r_{u}^{-4} \xi_{u} \sum_{i=-K}^{K} G_{u}^{2}(i)},
\end{gathered}
$$

where $r_{u}$ is the distance of the $u$ th interferer from the basestation of the intended user and $\xi_{u}$ is the lognormal shadowing

\footnotetext{
${ }^{12}$ Definitions of $G_{u}(i)$ are the same as in the CDMA analysis in Appendix A
}

of the $u$ th interferer with respect to the base-station of the intended user; $r_{0}$ and $\xi_{0}$ are the corresponding parameters of the intended user.

If a zero forcing equalizer is used, we must find the set of complex equalizer weights denoted by $\mathcal{C}=\left[C_{-K}, C_{-(K-1)}\right.$, $\left.\cdots, C_{0}, \cdots, C_{K-1}, C_{K}\right]$ which eliminate ISI. The coefficients can be found solving [13]

$$
\mathcal{F C}=\mathcal{A} \Rightarrow \mathcal{C}=\mathcal{F}^{-1} \mathcal{A},
$$

where

$$
\mathcal{A}=[0,0, \cdots, 1, \cdots, 0,0]^{T} .
$$

and the entries of matrix $\mathcal{F}$ are given by $\mathcal{F}_{i j}=F(|2 K-i-j|)$, where $i \in\{0,2 K\}$ and $j \in\{0,2 K\}$. Proceeding in this fashion, one can readily compute the multiple access interference (Details can be found in [17]).

\section{REFERENCES}

[1] IEEE Pers. Commun., Special issue on Wireless ATM, August 1996.

[2] D. Raychaudhuri and N.Wilson, "ATM Based Transport Architecture for Multiservices Wireless Personal Communication Networks", IEEE J. Select. Areas Commun., pp1401-1414, October 1994.

[3] A.S. Acampora and M.Naghshineh, "Control and Qualityof-Service Provisioning in High-Speed Microcellular Networks", IEEE Pers. Commun., pp. 36-43, vol.1, No.2, Second Quarter 1994.

[4] K.S. Gilhousen et al., "On the Capacity of a Cellular CDMA System”, IEEE Trans. Veh. Technol., pp. 303-312, vol.40, No.2, May 1991.

[5] K. Raith and J. Uddenfeldt, "Capacity of Digital Cellular TDMA Systems", IEEE Trans. on Veh. Technol., Vol.40, No.2, pp 323-332, May 1991.

[6] B. Gudmundson et al., "A Comparison of CDMA and TDMA Systems", Proc. of IEEE VTC '92, pp. 732-735, 1992.

[7] F. Borgonovo et al., "Capture Division Packet Access for Wireless Personal Communications", IEEE J. Select. Areas Commun., pp. 609-622, May 1996.

[8] F. Simpson and J.M. Holtzman, "Direct Sequence CDMA Power Control, Interleaving, and Coding”, IEEE J. Select. Areas Commun., pp 1085-1095, Sept. 1993.

[9] V.H.MacDonald, "The Cellular Concept" Bell Systems Tech. J, no.58, pp. 15-41, 1979.

[10] A.J. Viterbi et al., "Other-Cell Interference in Cellular Power-Controlled CDMA", IEEE Trans. Commun., Vol 42., No 2/3/4, February 1994.

[11] M. Zorzi, "On the Analytical Computation of the Interference Statistics with Applications to the Performance Evaluation of Mobile Radio Systems," IEEE Trans. Commun., vol. 45, pp. 103-110, Jan. 1997.

[12] W.C. Jakes, Jr., Ed., Microwave Mobile Communications, Wiley,NY, 1974.

[13] J.G. Proakis, Digital Communications, McGraw Hill Inc., Second Edition, 1989. 
[14] B. Glance and L.J. Greenstein, "Frequency-Selective Fading Effects in Digital Mobile Radio with Diversity Combining", IEEE Trans. Commun., Vol. COM- 31, No.9. pp 1085-1094, September 1983.

[15] D. Bertsekas and R. Gallager, Data Networks, Englewood Cliffs, N.J., Prentice-Hall, 1992.

[16] S. Lin and D.J. Costello Jr., Error Control Coding: fundamentals and applications, Englewood Cliffs, N.J., Prentice-Hall, 1983.

[17] S.V. Krishnamurthy et al., "A Capacity Comparison of TDMA and CDMA for Broadband Wireless Packet Access", Center for Wireless Communications Technical Report, University of California, San Diego 1997.

Srikanth V. Krishnamurthy (S'94 - M'00) received his Ph.D. degree in Electrical and Computer Engineering from the University of California, San Diego, La Jolla, in 1997. He is currently an Assistant Professor of Computer Science and Engineering at the University of California, Riverside. Prior to joining UC Riverside, from 1998 to 2000, he was a Research Staff Scientist at the Information Sciences Laboratory, HRL Laboratories, LLC, Malibu, CA, where he was leading various mobile wireless and satellite networking projects including the DARPA Next Generation Internet and the Small Unit Operations Projects. He is also a Co-Principal Investigator on the DARPA Fault Tolerant Networks Project at UC Riverside. His research interests span CDMA and TDMA technologies, medium access control for satellite and wireless networks, routing and multicasting in wireless networks, power management and the use of smart antennas in wireless networks, quality-of-service for the Internet and security. He was the Technical Co-Chair for the Workshop on Satellite Broadband Information Services (WOSBIS) held in conjunction with Globecom in 1999 and has been on the technical program committees for IEEE INFOCOM, ACM Mobihoc and IEEE ICC. He was also the local arrangements chair for ACM Mobihoc 2001 and IEEE ICNP 2001. E-Mail: krish@cs.ucr.edu

Anthony S. Acampora (S'68-M'68-SM'86-F' 88) is a Professor of Electrical and Computer Engineering at the University of California, San Diego, and is involved in numerous research projects addressing various issues at the leading edge of telecommunications networks, including the Internet, ATM, broadband wireless access, network management and dense wavelength division multiplexing. From 1995 through 1999, he was Director of UCSD's Center for Wireless Communications, responsible for an industrially funded research effort which included circuits, signal processing, smart antennas, basic communication theory, wireless telecommunication networks, infrastructure for wireless communications, and software for mobility. Since early 1997, he has been an advisor to the Board of Directors at Wireless Facilities, Inc., a San Diego company devoted to telecommunications outsourcing, and in March, 1998, he co-founded AirFiber Inc., a San Diegobased company involved in the development and marketing of broadband free space optical networking equipment for cellular and local exchange carriers. Prior to joining the faculty at UCSD in 1995, he was Professor of Electrical Engineering at Columbia University and Director of Center for Telecommunications Research, a national engineering research center. He joined the faculty at Columbia in 1988 following a 20-year career at AT\&T Bell Laboratories, most of which was spent in basic research where his research interests included radio and satellite communications, local and metropolitan area networks, packet switching, wireless access systems, and lightwave networks. His most recent position at Bell Labs was Director of the Transmission Technology Laboratory where he was responsible for a wide range of projects, including broadband networks, image communications and digital signal processing. At Columbia, he was involved in research and education programs concerning broadband networks, wireless access networks, network management, optical networks and

Michele Zorzi (S'89-M'95-SM'98) was born in Venice, Italy, in 1966. He received the Laurea Degree and the Ph.D. in Electrical Engineering from the University of Padova, Italy, in 1990 and 1994, respectively. During the Academic Year 1992/93, he was on leave at the University of California, San Diego (UCSD), attending graduate courses and doing research on multiple access in mobile radio networks. In 1993, he joined the faculty of the Dipartimento di Elettronica e Informazione, Politecnico di Milano, Italy. After spending three years with the Center for Wireless Communications at UCSD, in 1998 he joined the School of Engineering of the Università di Ferrara, Italy, where he is currently a Professor. His present research interests include performance evaluation in mobile communications systems, random access in mobile radio networks, and energy constrained communications protocols. ¿ Dr. Zorzi currently serves on the Editorial Boards of the IEEE Personal Communications Magazine, the ACM/URSI/Baltzer Journal of Wireless Networks, the IEEE Transactions on Wireless Communications, and the WILEY Journal of Wireless Communications and Mobile Computing. He is also guest editor for special issues in the IEEE Personal Communications Magazine (Energy Management in Personal Communications Systems) and the IEEE Journal on Selected Areas in Communications (Multi-media Network Radios). E-mail: zorzi@ing.unife.it 


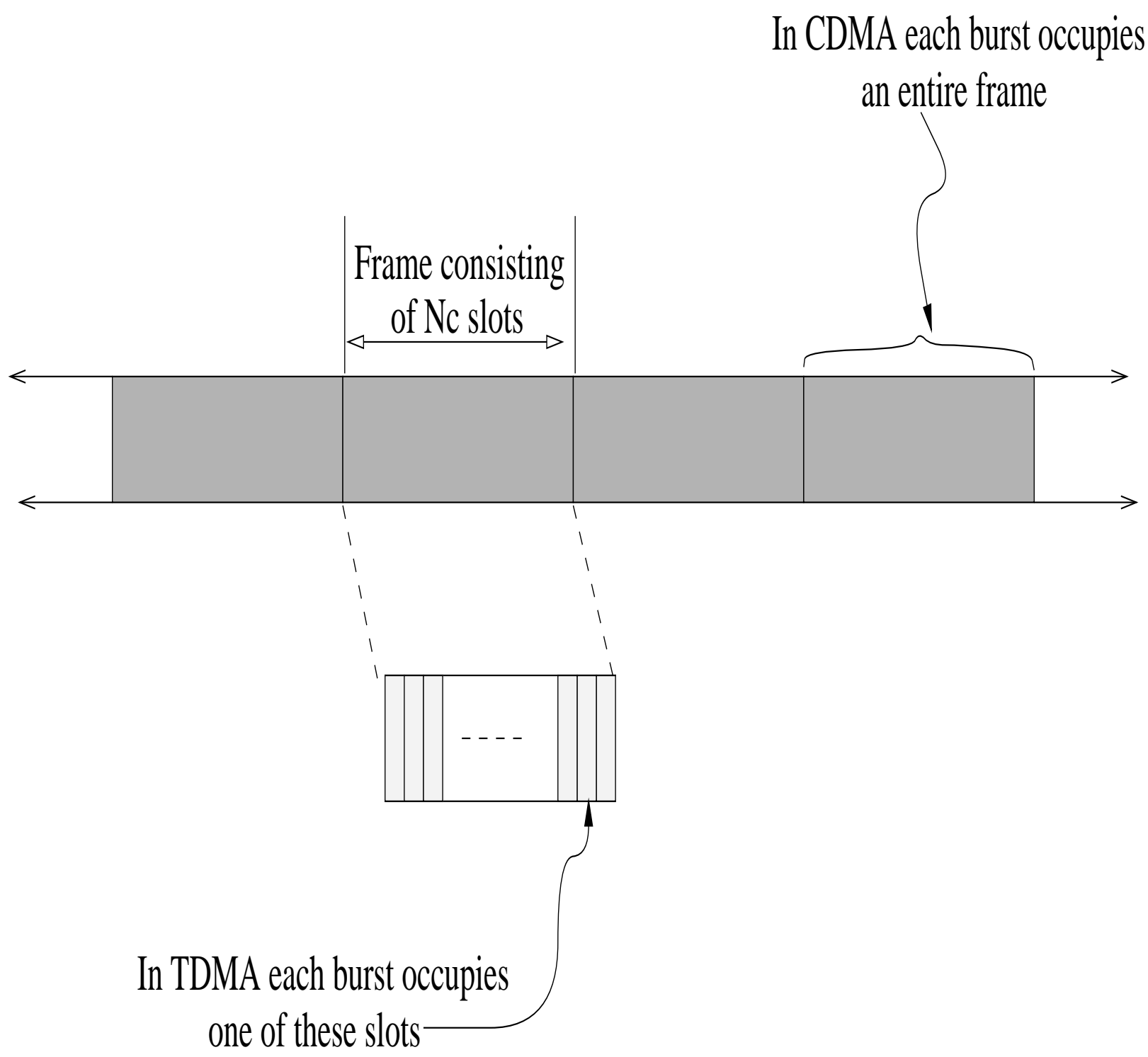

Fig. 1. Slot structures for the (a) CDMA and (b) TDMA schemes 


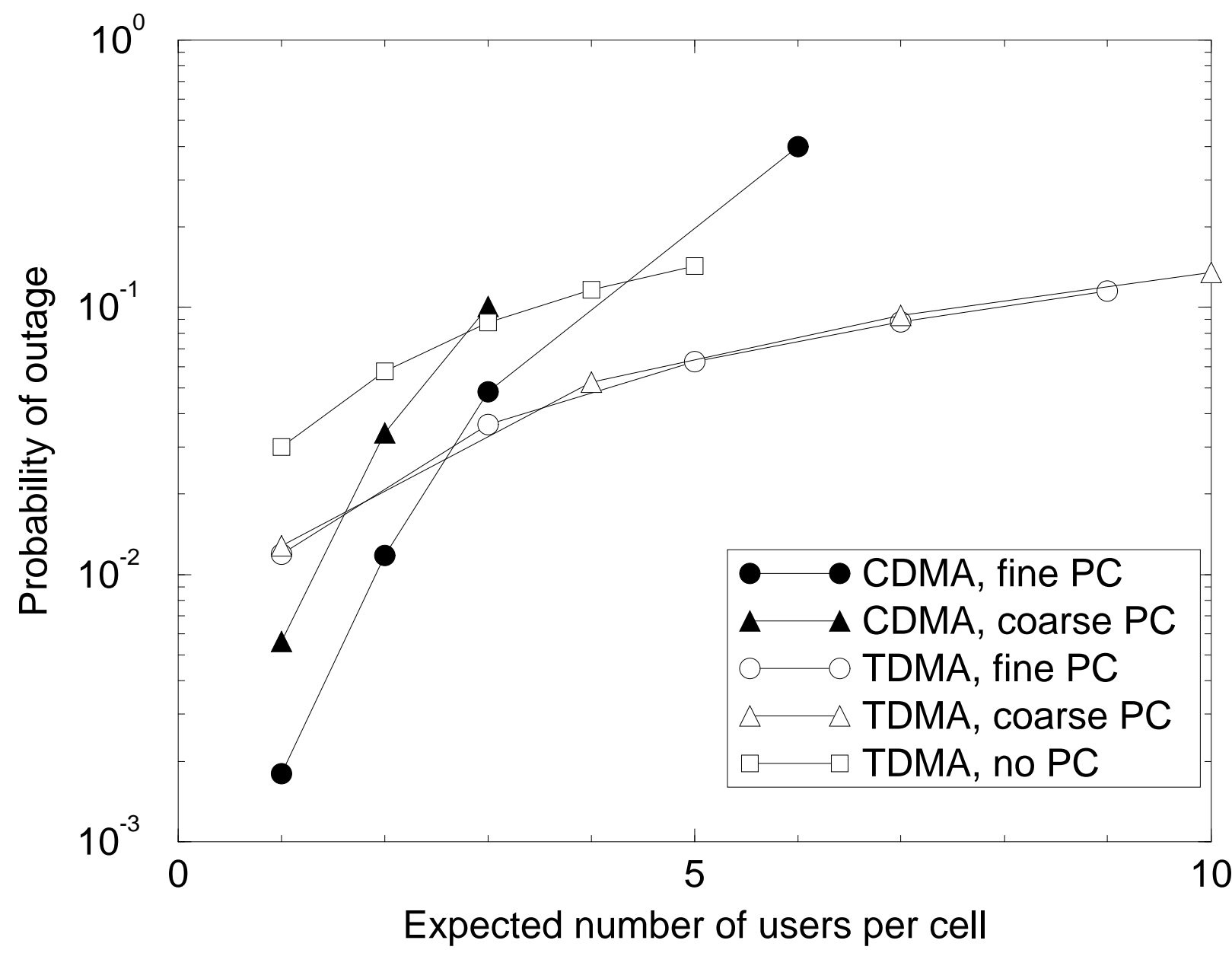

Fig. 2. CBR Traffic: Effects of Power Control. No coding, $N_{c}=50, T_{d}=2 T_{c}$. 


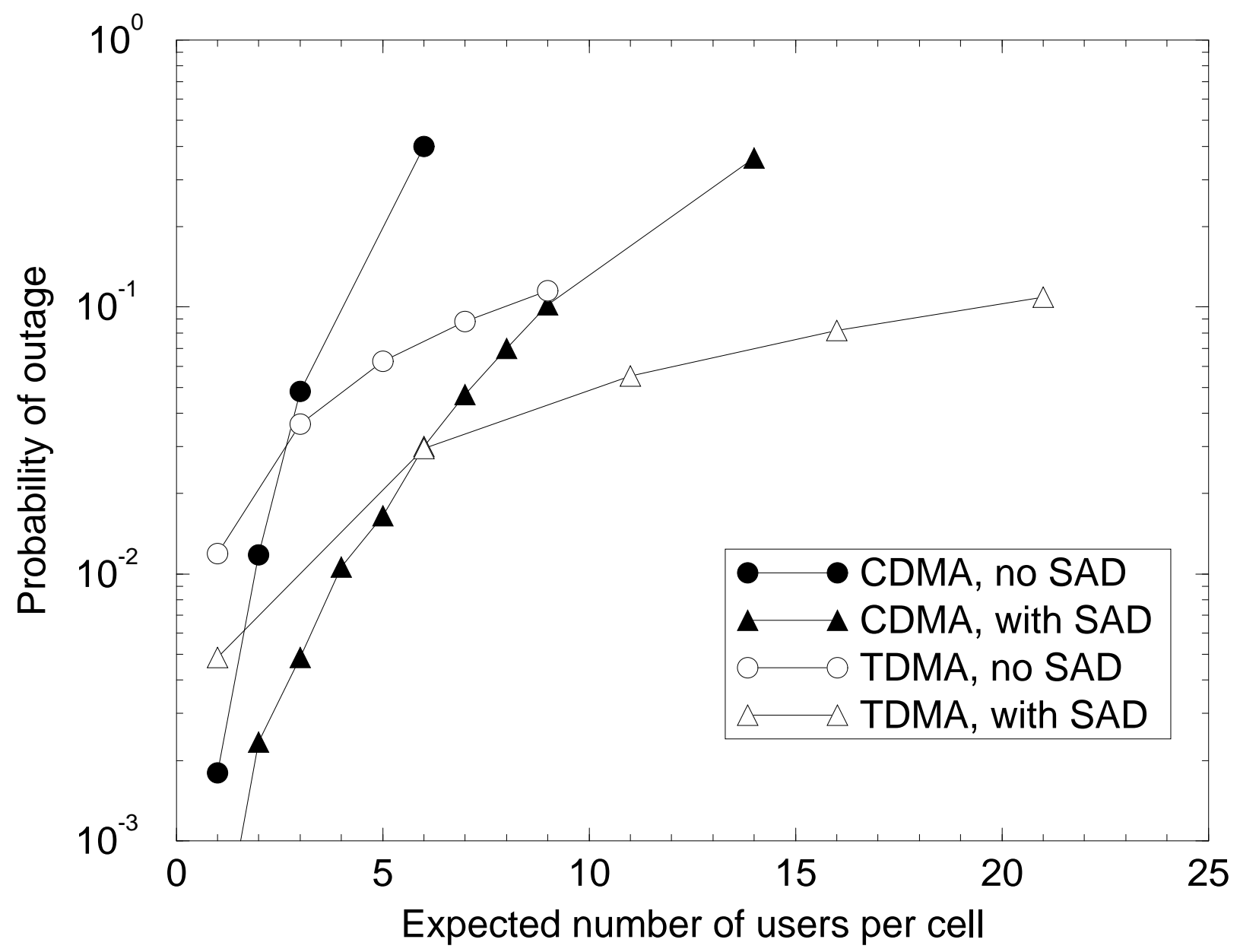

Fig. 3. CBR Traffic: Effects of Speech Activity Detection (SAD). No coding, $N_{c}=50, T_{d}=2 T_{c}$. 


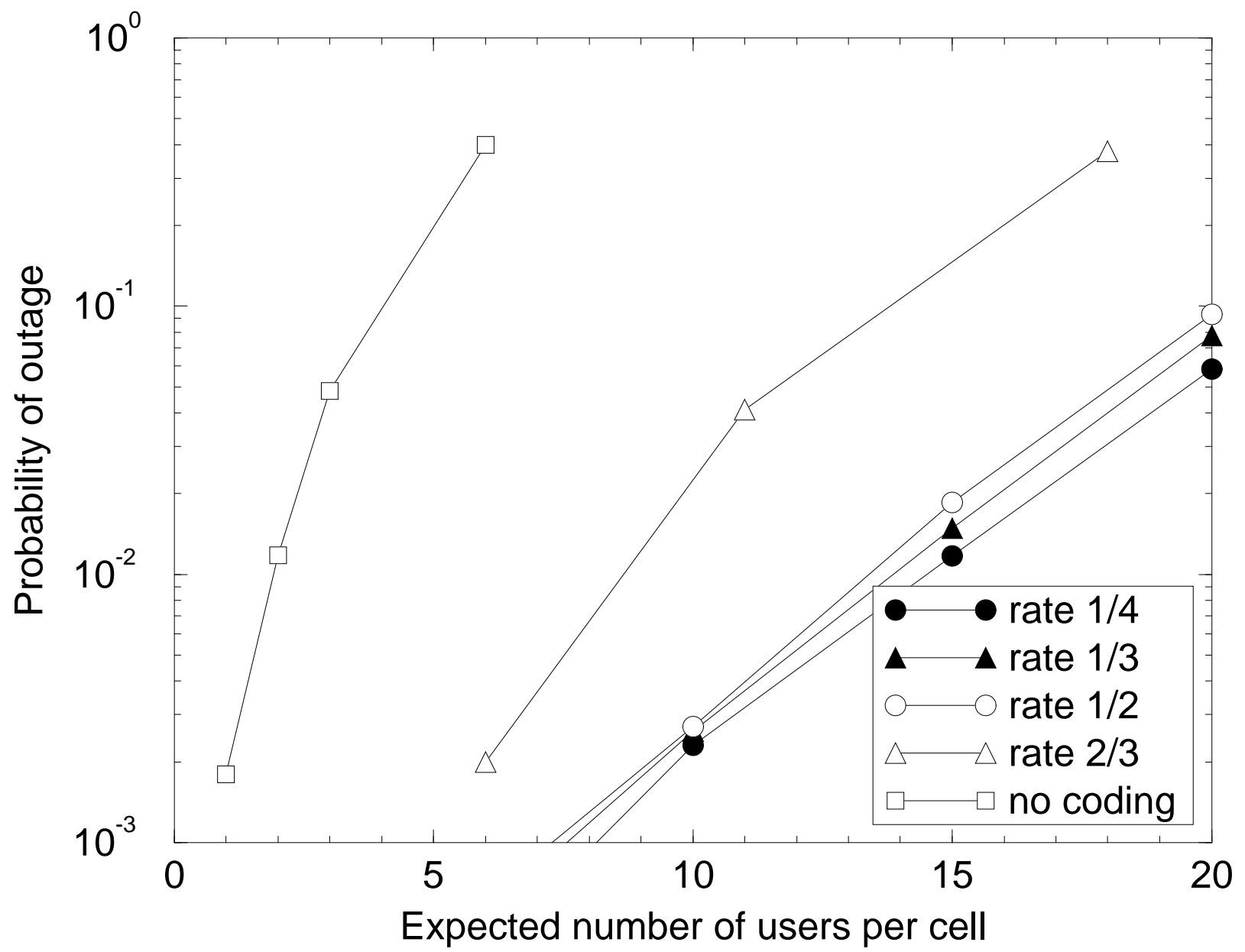

Fig. 4. CBR Traffic, CDMA: Performance of Different Codes. Fine power control, $N_{c}=50, T_{d}=2 T_{c}$, no SAD. 


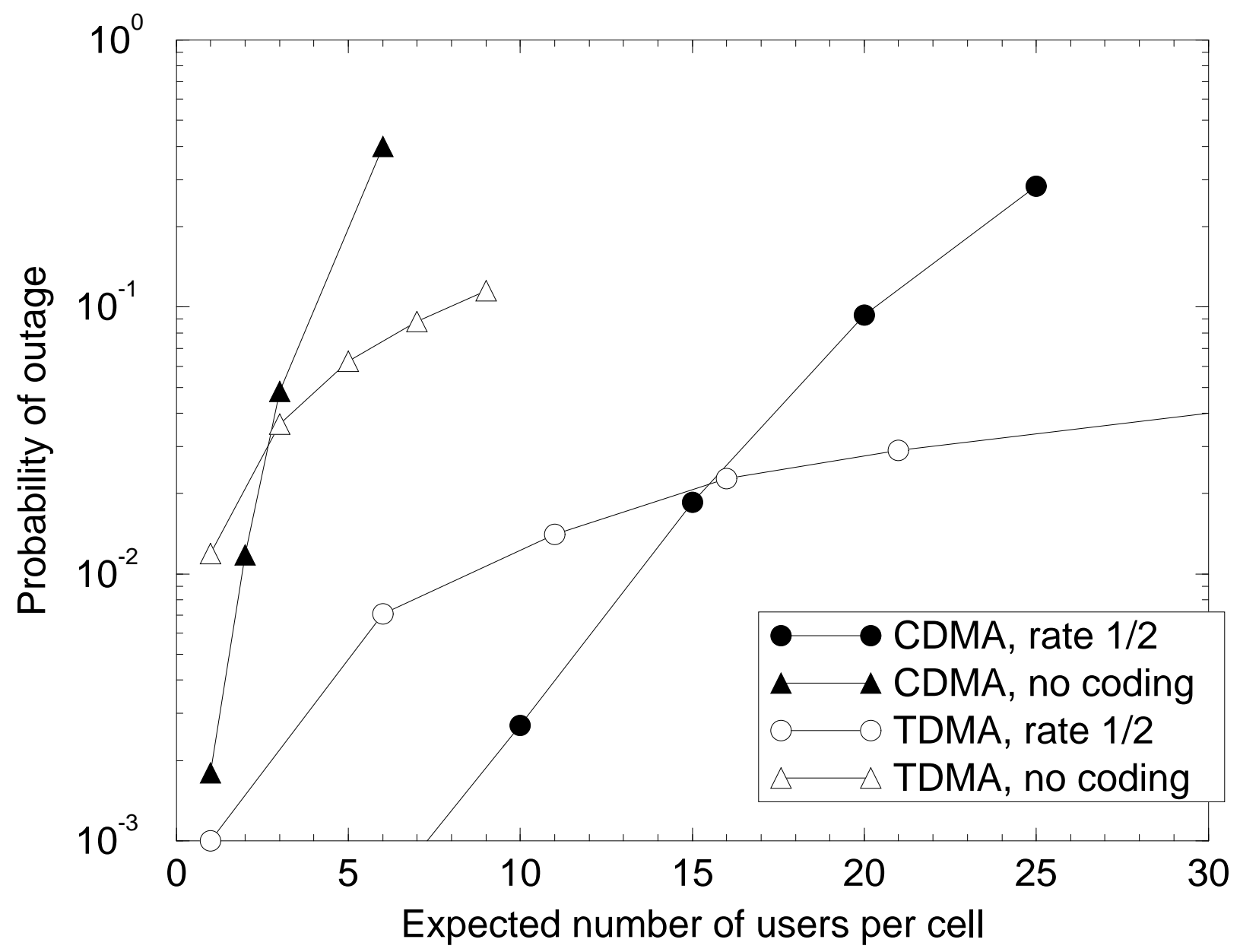

Fig. 5. CBR Traffic: Comparison of CDMA and TDMA schemes. Fine power control, $N_{c}=50, T_{d}=2 T_{c}$, no SAD. 


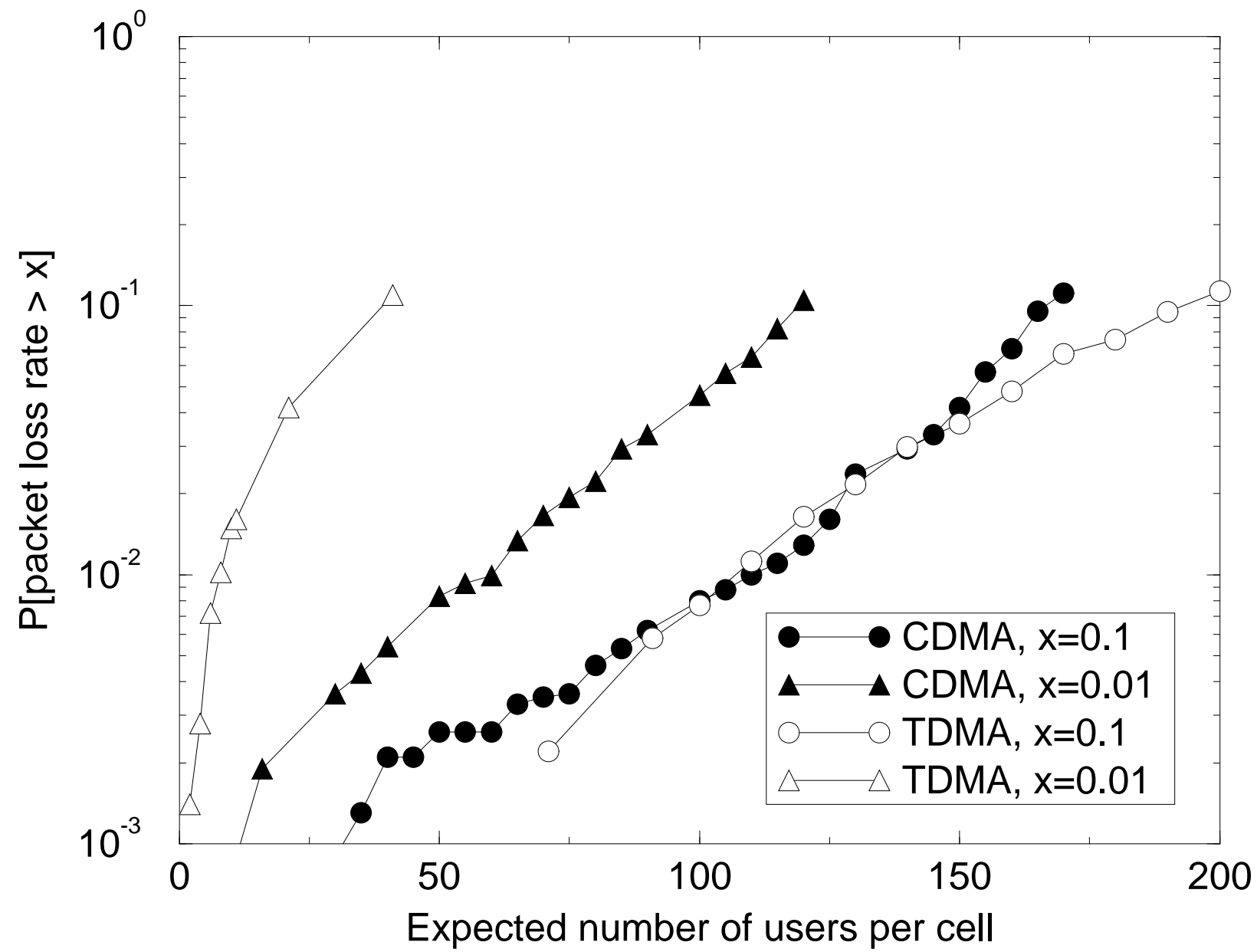

Fig. 6. Packet Traffic with no queueing allowed: Comparison of CDMA and TDMA schemes. Fine power control, rate $1 / 2$ code, $N_{c}=50, T_{d}=2 T_{c}, q=0.1$. 


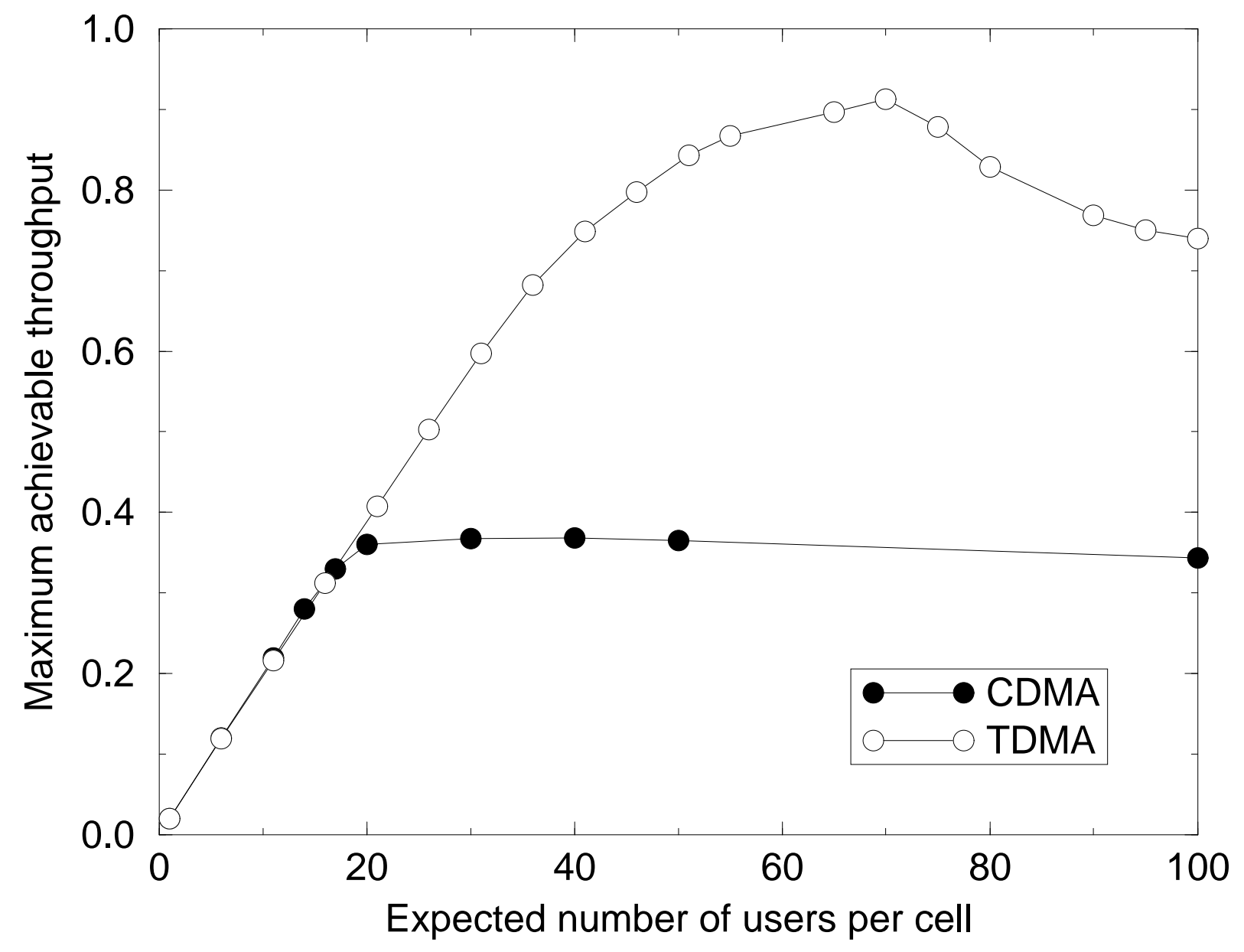

Fig. 7. Maximum Saturation throughput: CDMA and TDMA. Fine power control, rate $1 / 2$ code, $N_{c}=50, T_{d}=2 T_{c}$. 


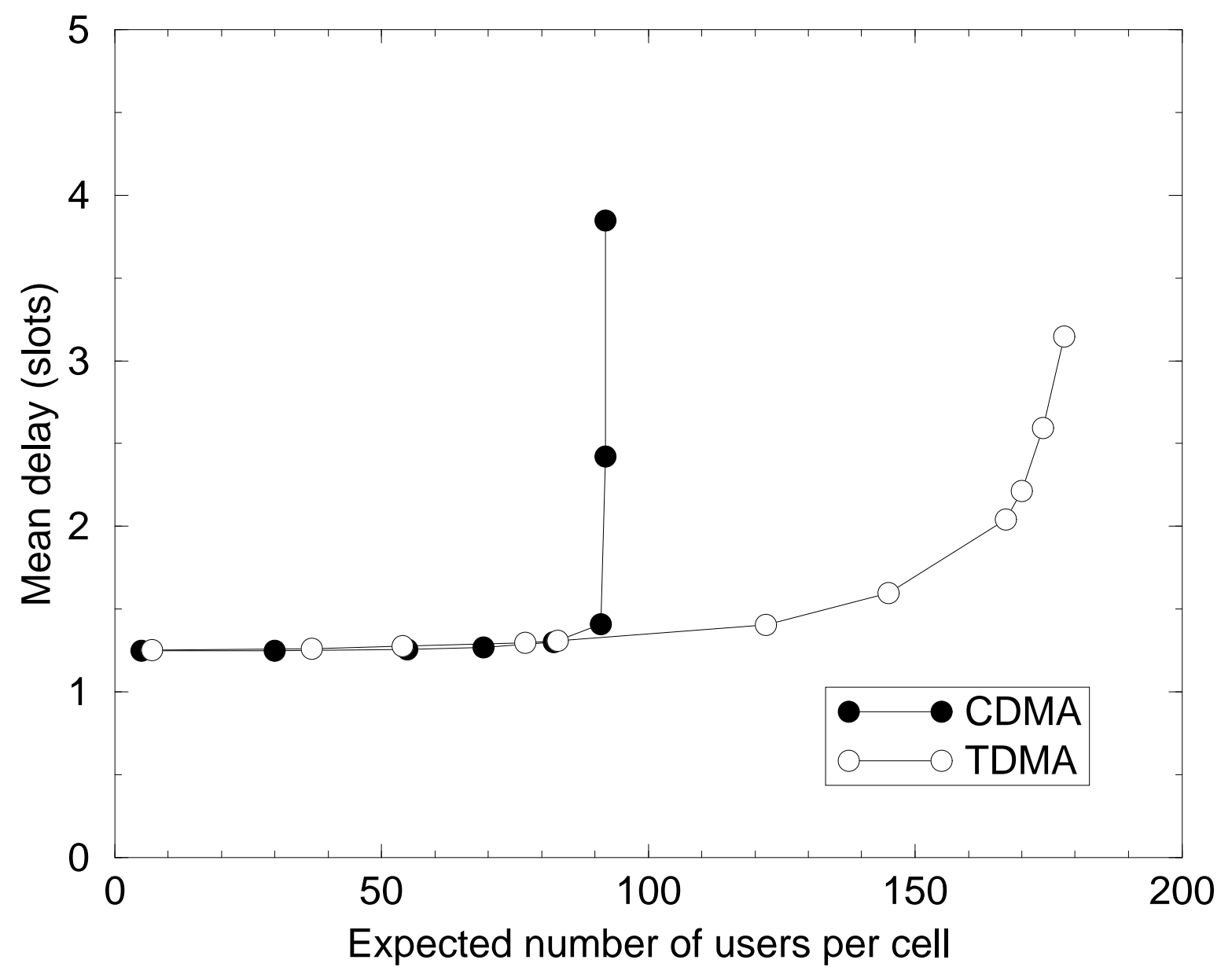

Fig. 8. Packet Traffic with mean delay constraints. Fine power control, rate $1 / 2$ code, $N_{c}=50, T_{d}=2 T_{c}$ 